\title{
Lateral orbitofrontal gray matter abnormalities in subjects with problematic smartphone use
}

\author{
DEOKJONG LEE ${ }^{1,2}$, KEE NAMKOONG $^{2,3}$, JUNGHAN LEE $^{2,3}$, BYUNG OOK LEE $^{1,2}$ and YOUNG-CHUL JUNG ${ }^{2,3 *}$ \\ ${ }^{1}$ Department of Psychiatry, Ilsan Hospital, National Health Insurance Corporation, Goyang, Republic of Korea \\ ${ }^{2}$ Institute of Behavioral Science in Medicine, Yonsei University College of Medicine, Seoul, Republic of Korea \\ ${ }^{3}$ Department of Psychiatry, Yonsei University College of Medicine, Seoul, Republic of Korea
}

(Received: March 26, 2019; revised manuscript received: July 13, 2019; accepted: August 17, 2019)

\begin{abstract}
Background and aims: Smartphone use is becoming commonplace and exerting adequate control over smartphone use has become an important mental health issue. Little is known about the neurobiology underlying problematic smartphone use. We hypothesized that structural abnormalities in the fronto-cingulate brain region could be implicated in problematic smartphone use, similar to that has been reported for Internet gaming disorder and Internet addiction. This study investigated fronto-cingulate gray matter abnormalities in problematic smartphone users, particularly those who spend time on social networking platforms. Methods: The study included 39 problematic smartphone users with excessive use of social networking platforms via smartphone and 49 normal control male and female smartphone users. We conducted voxel-based morphometric analysis with diffeomorphic anatomical registration using an exponentiated Lie algebra algorithm. Region of interest analysis was performed on the fronto-cingulate region to identify whether gray matter volume (GMV) differed between the two groups. Results: Problematic smartphone users had significantly smaller GMV in the right lateral orbitofrontal cortex (OFC) than healthy controls, and there were significant negative correlations between GMV in the right lateral OFC and the Smartphone Addiction Proneness Scale (SAPS) score, including the SAPS tolerance subscale. Conclusions: These results suggest that lateral orbitofrontal gray matter abnormalities are implicated in problematic smartphone use, especially in social networking platform overuse. Small GMV in the lateral OFC was correlated with an increasing tendency to be immersed in smartphone use. Our results suggest that orbitofrontal gray matter abnormalities affect regulatory control over previously reinforced behaviors and may underlie problematic smartphone use.
\end{abstract}

Keywords: orbitofrontal cortex, gray matter volume, problematic smartphone use

\section{INTRODUCTION}

Smartphones have come into widespread, daily use for most people. They provide an environment that is continuously connected to the Internet, which facilitates contact with online-related activities. This environment has been suggested as a risk factor for excessive immersion in some online activities (Stald et al., 2014). Many people display "problematic smartphone user" behaviors and fail to regulate their excessive smartphone use, despite negative psychosocial consequences (Long et al., 2016; Lopez-Fernandez, Honrubia-Serrano, Freixa-Blanxart, \& Gibson, 2014). However, little is known about the core features of problematic smartphone use and its underlying neurobiological mechanisms. Previous studies indicate that comorbid psychiatric conditions, such as depression and anxiety, are closely related to problematic smartphone use (Elhai, Dvorak, Levine, \& Hall, 2017; Elhai, Tiamiyu, \& Weeks, 2018). Some studies focused on the role of psychological factors, such as empathy, life satisfaction (Lachmann et al., 2018), and fear of missing out (Wolniewicz, Tiamiyu, Weeks, \& Elhai, 2018). Other studies suggest that problematic smartphone use may correspond to behavioral addictions, such as Internet gaming disorder and Internet addiction (Salehan \& Negahban, 2013). Previous studies reported that problematic smartphone users exhibit craving, salience, and loss of control, which are related to their psychological dependence on online smartphone activities (Chen, Liang, Mai, Zhong, \& Qu, 2016; Sapacz, Rockman, \& Clark, 2016; Wegmann, Stodt, \& Brand, 2018). Several studies suggested that problematic smartphone use is closely related to the addictive use of social networking platforms (Salehan \& Negahban, 2013; Sha, Sariyska, Riedl, Lachmann, \& Montag, 2019). It has been suggested that the formation and reinforcement of habitual social networking platform use could lead to Internet communication addiction (Turel \& Serenko, 2012). Smartphones provide a readily available technology that likely facilitates the progression to addictive use of social media.

\footnotetext{
* Corresponding author: Young-Chul Jung, MD, PhD; Department of Psychiatry, Yonsei University College of Medicine, 03722 Yonsei-ro, Seodaemun-gu, Seoul 120 752, Republic of Korea; Phone: +82 22228 1620; Fax: +82 022313 0891; E-mail: eugenejung@yuhs.ac
}

This is an open-access article distributed under the terms of the Creative Commons Attribution-NonCommercial 4.0 International License, which permits unrestricted use, distribution, and reproduction in any medium for non-commercial purposes, provided the original author and source are credited, a link to the CC License is provided, and changes - if any - are indicated. 
The neurobiology of problematic smartphone use has not been completely mapped, although recent studies have identified various structural and functional brain abnormalities in Internet gaming disorder and Internet addiction. These abnormalities include gray matter volume (GMV) deficiencies in the anterior cingulate and the orbitofrontal and dorsolateral prefrontal cortex (Lee, Namkoong, Lee, \& Jung, 2018; Yao et al., 2017). These fronto-cingulate areas are associated with exerting executive control (Yuan \& Raz, 2014), which is the ability to use higher-order cognitive functions appropriately to perform goal-directed behaviors (Miller \& Wallis, 2009). Exerting appropriate executive control is believed to be important in balancing goaldirected actions and habitual behaviors (Jahanshahi, Obeso, Rothwell, \& Obeso, 2015). Habitual behaviors require less executive control than goal-directed actions (Dalley, Cardinal, \& Robbins, 2004; Müller et al., 2007). Addiction involves a process in which goal-directed actions are progressively shifted into habitual behaviors (Everitt, Dickinson, \& Robbins, 2001). Previously presented disease models of Internet gaming disorder and Internet addiction have emphasized the role of executive control dysfunction (Brand, Young, Laier, Wölfling, \& Potenza, 2016; Dong \& Potenza, 2014). Some studies suggest that fronto-cingulate GMV deficiencies are involved in problematic smartphone use. A study of subjects who had self-reported addiction symptoms to smartphone use showed that they had smaller GMVs in the frontal regions and left anterior cingulate cortex than healthy control users (Wang et al., 2016). Smartphone users who had higher tendencies toward addiction to Internet communication applications also had smaller GMVs in the subgenual anterior cingulate cortex (Montag et al., 2018). Therefore, we hypothesized that low GMVs in executive control-related brain regions, including the fronto-cingulate area, could be associated with the pathophysiology of problematic smartphone use. This hypothesis is consistent with neurophysiological evidence suggesting that dysfunctional executive control, such as attention and inhibitory control, is related to problematic smartphone use (Chen et al., 2016; Hadar et al., 2017).

This study aimed to identify the neurobiological basis of problematic smartphone use. Because problematic smartphone use is closely associated with social networking addiction, this study focused on problematic smartphone users with excessive social networking platform use via smartphone. We examined the gray matter properties in the fronto-cingulate area in problematic smartphone users and healthy controls by performing voxel-based morphometry (VBM) analysis to compare GMVs between the two groups. Then, correlation analyses were performed to analyze whether GMVs of problematic smartphone users were related to their clinical features.

\section{MATERIALS AND METHODS}

\section{Participants}

Participants were recruited via online advertisements, flyers, and word of mouth. Initially, 94 subjects $(65$ males and 29 females) were recruited. Participants were screened for excessive smartphone use with the Korean Smartphone Addiction Proneness Scale (SAPS; Kim et al., 2012; Kim, Lee, Lee, Nam, \& Chung, 2014). Subjects were classified as excessive smartphone users if their total SAPS score exceeded 40, or if their subscale score exceeded 14 for disturbance of adaptive function. Otherwise, subjects were classified as healthy controls. This study was aimed at those who use smartphones for Internet communication and social networks, so participants who use smartphones primarily for other purposes such as gaming were excluded. All subjects underwent the Structured Clinical Interview from the Diagnostic and Statistical Manual of Mental Disorders, Fourth Edition (First, Spitzer, Gibbon, \& Williams, 1995) to assess major psychiatric illness, and the Korean version of the Wechsler Adult Intelligence Scale IV to measure intelligence quotient (IQ; Wechsler, 2008). Exclusion criteria for all subjects were: major psychiatric disorder (other than Internet addiction or problematic smartphone use), mental retardation, neurological or medical illness, and contraindications on the magnetic resonance imaging (MRI) scan. Finally, 88 subjects were included in this study: 29 males with excessive smartphone use, 10 females with excessive smartphone use, 32 healthy males, and 17 healthy females, all right-handed, aged between 16 and 27 years (mean: $22.6 \pm 2.4$ years). All subjects included in this study did not receive psychiatric treatment, including psychopharmacology.

\section{Psychometric measures}

The SAPS test was developed by the Korean National Information Society Agency to evaluate problematic smartphone use (Kim et al., 2014). The SAPS scale consists of a total of 15 items rated by a 4-point Likert scale ( $1=$ strongly disagree; 2 = disagree; 3 =agree; $4=$ strongly agree), so the score can range from 15 to 60 . The SAPS scale includes the following four subscales: disturbance of adaptive functions, virtual life orientation, withdrawal, and tolerance. Subjects with the following SAPS scores were classified as high-risk smartphone users: (a) total SAPS score of $\geq 44$ or (b) disturbance of adaptive functions, withdrawal, and tolerance subscale scores of $\geq 15, \geq 13$, and $\geq 13$, respectively. Subjects with the following SAPS scores were classified as potentially at-risk smartphone users: (a) total SAPS total score of $\geq 40$ or $\leq 43$ or (b) disturbance of adaptive functions subscale score of $\geq 14$. Those not belonging to the high-risk or potentially at-risk users were classified as normal smartphone users. The original Cronbach's $\alpha$ value of the SAPS score was .814, whereas the Cronbach's $\alpha$ value of the SAPS score in this study was 934. During scale development, each of the SAPS subscales (disturbance of adaptive functions, virtual life orientation, withdrawal, and tolerance) had a Cronbach's $\alpha$ value of $.832, .687, .776$, and .780 , respectively. In this study, each of the SAPS subscales (disturbance of adaptive functions, virtual life orientation, withdrawal, and tolerance) had a Cronbach's $\alpha$ value of $.874, .473, .855$, and .885 , respectively. Thus, three SAPS subscales exhibited adequate internal consistency (except for virtual life orientation).

The Internet Addiction Test (IAT; Young, 1998) was administered to assess Internet addiction status. The Barratt 
Impulsiveness Scale version 11 (BIS-11; Patton \& Stanford, 1995) was administered to test impulsivity. The Cronbach's $\alpha$ values in this study for IAT and BIS-11 were .94 and .80, respectively. To assess comorbid psychiatric conditions of depression, anxiety, and alcohol use disorder, all subjects took the Beck Depression Inventory (BDI; Beck, Steer, \& Brown, 1996), the Beck Anxiety Inventory (BAI; Beck, Epstein, Brown, \& Steer, 1988), and the Alcohol Use Disorder Identification Test (AUDIT; Saunders, Aasland, Babor, de la Fuente, \& Grant, 1993), respectively. The Cronbach's $\alpha$ values in this study for BDI, BAI, and AUDIT were $.80, .81$, and .75 , respectively.

\section{Structural image acquisition and preprocessing}

Brain MRI was conducted using a 3T Siemens Magnetom MRI scanner (Erlangen, Germany) equipped with an eightchannel head coil. A 3D structural MRI data set was obtained for each subject through a T-1 weighted spoiled gradient echo sequence $(\mathrm{TE}=2.19 \mathrm{~ms}$, $\mathrm{TR}=1,780 \mathrm{~ms}$, flip angle $=9^{\circ}$, field of view $=256 \mathrm{~mm}$, matrix $=256 \times 256$, transversal slice thickness $=1 \mathrm{~mm}$ ). Structural brain images were analyzed with MATLAB 8.5 (MathWorks, Natick, MA, USA) and SPM8 (Wellcome Department of Imaging Neuroscience, UK). All preprocessing steps were performed according to the standardized procedure (Ashburner, 2010). First, structural images were aligned along the anteriorposterior commissure line, and the anterior commissure of each image was matched to the origin position. Then, the structural images were segmented into gray matter, white matter, and cerebrospinal fluid probability maps using a Bayesian image segmentation algorithm. The brain tissue probability maps for each subject were used for intersubject alignment. We used diffeomorphic anatomical registration with an exponentiated Lie algebra algorithm (DARTEL) to increase the accuracy of intersubject alignment by modeling the shape of each brain using a host of parameters (Ashburner, 2007). DARTEL processing includes generating the flow fields that encode deformations and creating the study-specific templates for all subjects. Gray matter images for each subject were warped to the study-specific template and normalized into standard Montreal Neurological Institute $(\mathrm{MNI})$ space. The images were then resampled to a $1.5 \times 1.5 \times 1.5 \mathrm{~mm}^{3}$ voxel size. This spatial normalization step involved Jacobian modulation to preserve regional volume values. Finally, the warped, normalized, and modulated gray matter images were smoothed using an $8-\mathrm{mm}$ full-width at half maximum kernel.

\section{Statistical analysis}

Statistical analyses were performed using the Statistical Package for the Social Sciences (SPSS) version 24.0 (SPSS Inc., Chicago, IL, USA). Differences with $p$ values $<.05$ were deemed to be statistically significant. To compare demographic data (clinical characteristics of excessive smartphone users and healthy controls), we employed independent $t$-tests and $\chi^{2}$ tests. To evaluate group differences in GMV, we used an analysis of covariance model at each voxel. The age, sex, and intracranial volume of each subject were entered as covariates. Intracranial volumes were calculated from the sum of gray matter, white matter, and cerebrospinal fluid volume.

Initially, region of interest (ROI) analysis was performed to explore the group differences in GMV. The frontocingulate regions (superior/middle/inferior frontal gyrus and cingulate cortex) were set as ROI, which was defined by the Individual Brain Atlas Statistical Parametric Mapping (Alemán-Gómez, 2006) provided by the Wake Forest University Pickatlas toolbox (Maldjian, Laurienti, Kraft, \& Burdette, 2003). Subsequently, exploratory analysis of the whole brain was performed to explore the region outside of ROI. Statistical inferences were set via cluster extent-based thresholding (Friston, Worsley, Frackowiak, Mazziotta, \& Evans, 1994). Initially, a voxel-wise cluster-defining threshold of uncorrected $p<.001$ was applied. Then, we reported significant clusters with a cluster-level extent threshold of false-wise error rate corrected $p<.05$.

We conducted partial correlation analyses to verify that GMV correlated with clinical variables (SAPS and BIS). The mean GMV of clusters with group differences was extracted using the MarsBaR toolbox (http://marsbar. sourceforge.net/). The age, sex, and intracranial volume of each subject were entered as covariates. In subsequent correlation analyses, parameters related to comorbid conditions (BDI, BAI, and AUDIT) were added as covariates.

\section{Ethics}

This study was carried out under the guidelines for the use of human participants established by the Institutional Review Board at Yonsei University. All protocols for this study were approved by the Institutional Review Board at Severance Hospital, Yonsei University. Written informed consent was obtained from all participants before enrollment.

\section{RESULTS}

\section{Demographic and clinical characteristics of study subjects}

Healthy control subjects and subjects with problematic smartphone use were matched by age, sex, and IQ (Table 1). Subjects with problematic smartphone use spent significantly more daily time using their smartphone than healthy controls $(p<.001)$. We calculated Bonferroni's correction $(p<.05 / 4)$ to adjust for multiple comparisons (four subdomains of usage goals: gaming, entertainment, social communication platforms, and Internet surfing) and found that the time spent on social communication applications significantly differed between the two groups $(p<.001)$. Problematic smartphone users scored significantly higher on SAPS and SAPS subscales $(p<.001$, respectively), and IAT and BIS ( $p=.001$, respectively) than healthy control subjects. Problematic smartphone users scored significantly higher on tests for depression (BDI), anxiety (BAI), and alcohol-related problems (AUDIT; $p=.026, p=.006$, and $p=.006$, respectively) than healthy controls. 
Table 1. Demographics and clinical variables of study participants

\begin{tabular}{|c|c|c|c|c|}
\hline & $\begin{array}{l}\text { Problematic smartphone } \\
\text { users }(n=39)\end{array}$ & Healthy controls $(n=49)$ & & \\
\hline & Mean $(S D)$ & Mean $(S D)$ & Test & $p$ value \\
\hline Age (years) & $22.9(2.2)$ & $22.4(2.7)$ & $t=0.921$ & .360 \\
\hline Sex $[$ male; $n(\%)]$ & $29(74.4)$ & $32(65.3)$ & $\chi^{2}=0.837$ & .360 \\
\hline Full-scale $\mathrm{IQ}^{\mathrm{a}}$ & $110.3(12.4)$ & $109.5(11.0)$ & $t=0.338$ & .737 \\
\hline Smartphone addiction & $45.0(4.8)$ & $28.9(6.4)$ & $t=13.434$ & $<.001$ \\
\hline \multicolumn{5}{|l|}{ Proneness scale total } \\
\hline Disturbance of adaptive functions & $15.6(1.7)$ & $9.4(2.4)$ & $t=14.115$ & $<.001$ \\
\hline Virtual life orientation & $4.1(1.2)$ & $2.8(0.9)$ & $t=5.838$ & $<.001$ \\
\hline Withdrawal & $11.7(2.2)$ & $8.1(2.6)$ & $t=6.961$ & $<.001$ \\
\hline Tolerance & $13.6(1.5)$ & $8.7(2.7)$ & $t=10.606$ & $<.001$ \\
\hline Duration of smartphone use per day (hr) & $6.8(2.0)$ & $2.5(1.2)$ & $t=11.976$ & $<.001$ \\
\hline Gaming & $0.5(1.0)$ & $0.1(0.4)$ & $t=2.560$ & .014 \\
\hline Entertainment & $1.0(1.0)$ & $0.6(0.7)$ & $t=2.283$ & .025 \\
\hline Social communication platform & $4.6(1.6)$ & $1.5(0.9)$ & $t=10.645$ & $<.001$ \\
\hline Internet surfing & $0.7(0.9)$ & $0.3(0.5)$ & $t=2.239$ & .029 \\
\hline Internet Addiction Test & $48.7(14.1)$ & $36.9(18.2)$ & $t=3.336$ & .001 \\
\hline Beck Depression Inventory & $8.4(4.8)$ & $6.0(5.0)$ & $t=2.270$ & .026 \\
\hline Beck Anxiety Inventory & $8.3(6.0)$ & $5.1(4.6)$ & $t=2.840$ & .006 \\
\hline Alcohol Use Disorder Identification Test & $10.4(4.9)$ & $7.5(4.5)$ & $t=2.834$ & .006 \\
\hline Barratt Impulsivity Scale & $53.8(7.5)$ & $48.2(7.3)$ & $t=3.523$ & .001 \\
\hline
\end{tabular}

Note. IQ: intelligence quotient; $S D$ : standard deviation.

${ }^{\mathrm{a}} \mathrm{IQ}$ was assessed using the Wechsler Adult Intelligence Scale.

\section{Voxel-based morphometry (VBM) results}

Compared to healthy control subjects, problematic smartphone users exhibited smaller GMV in the right orbitofrontal cortex (OFC; Figure 1A). These clusters remained significant after comorbid conditions (BDI, BAI, and AUDIT) had been included as covariates $(p<.001)$. The GMV differences in the OFC were significant when the two groups were stratified with respect to sex (Figure 1B). The problematic smartphone users did not display significantly larger GMV than healthy controls in any brain region. There were no significant clusters with group differences, outside of the ROI.

The correlation analysis showed that smaller GMV in the right OFC was significantly correlated with higher total SAPS score $(r=-.449, p=.006$; Figure 2A). These correlations remained significant after comorbid conditions (BDI, BAI, and AUDIT) had been included as covariates $(p=.035)$. We calculated Bonferroni's correction $(p<.05 / 4)$

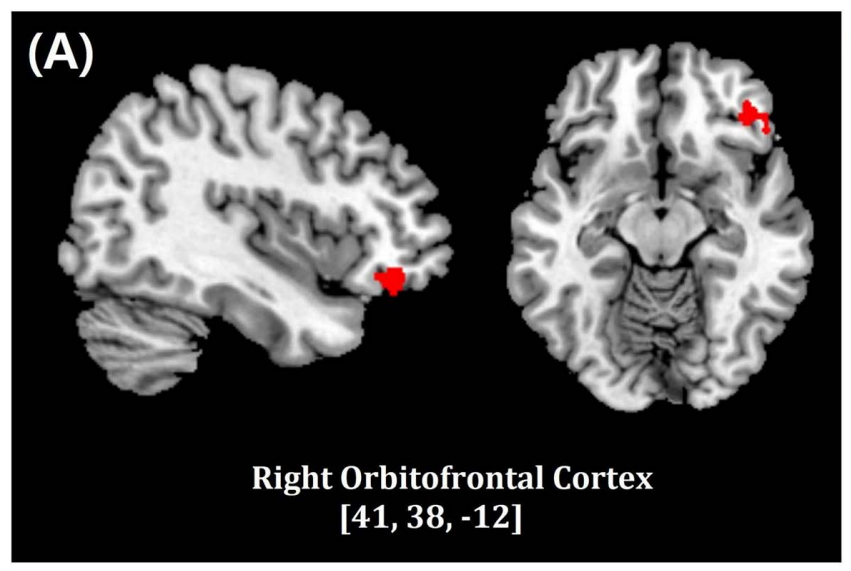

(B)

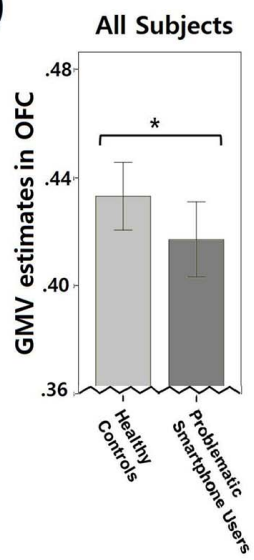

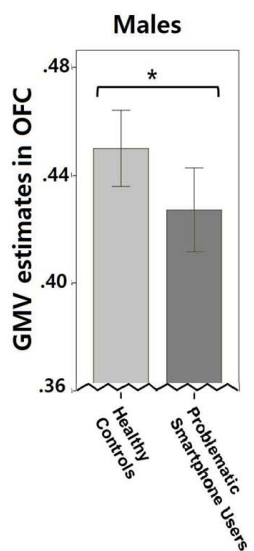

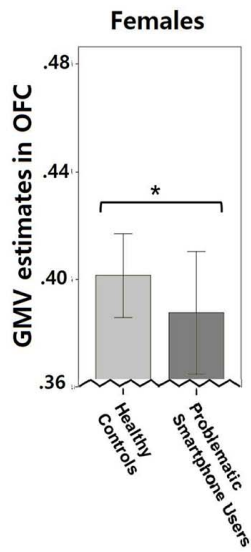

Figure 1. Voxel-based morphometric analysis of the fronto-cingulate region as the region of interest (ROI). Statistical inferences were thresholded using an uncorrected $p$ value height threshold of .001 in conjunction with an extent threshold correction of false-wise error rate of $p<.05$. Coordinates indicate the locations of the brain slices according to the Montreal Neurological Institute system. (A) Subjects with problematic smartphone use showed significantly smaller gray matter volume than healthy controls in the right lateral orbitofrontal cortex (OFC). (B) Significant gray matter volume (GMV) differences were observed in the OFC when the groups were stratified with respect to sex (male, $p=.001$; female, $p=.007$ ) 

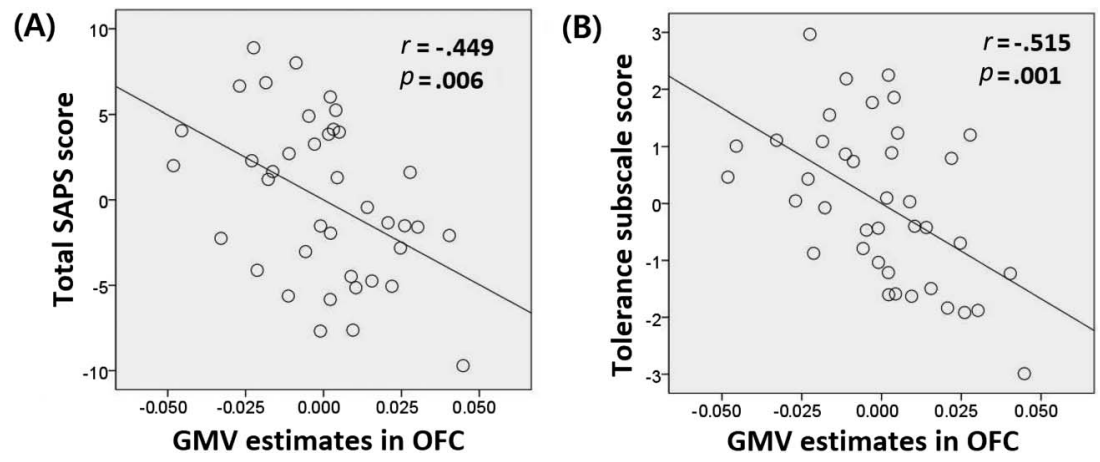

Figure 2. Correlation analysis of the mean gray matter volume (GMV) value for clusters in the right lateral orbitofrontal cortex (OFC) and clinical variables for subjects with problematic smartphone use $(n=39)$. Partial correlation analysis was performed to control for covariates (age, sex, and intracranial volume). To depict partial correlation, linear regression was used to regress variables onto covariates. Calculated non-standardized residuals were used to generate scatter plots. (A) Smaller GMV in the right OFC correlated significantly with higher total SAPS score $(r=-.449, p=.006)$. (B) Smaller GMV in the right OFC correlated significantly with higher SAPS tolerance subscales scores $(r=-.515, p=.001)$

to adjust for multiple comparisons (four subscales of the SAPS) and found that smaller GMV in the right OFC significantly correlated with higher SAPS tolerance subscale score $(r=-.515, p=.001$; Figure 2B). These correlations remained significant after comorbid conditions (BDI, BAI, and AUDIT) had been included as covariates $(p=.008)$. Other correlation tests did not reach statistical significance.

\section{DISCUSSION}

This study analyzed the GMV of subjects who spend an excessive amount of time on social networking platforms through smartphones. Subjects with problematic smartphone use had smaller GMV in the right OFC than normal control subjects. This result supports our hypothesis that subjects with problematic smartphone use could have gray matter abnormalities in the fronto-cingulate region. This study results were not consistent with those of previous studies on gray matter abnormalities, which identified structural brain abnormalities in problematic smartphone users such as small GMV in the anterior cingulate cortex (Montag et al., 2018; Wang et al., 2016). However, our identification of gray matter abnormalities in the OFC of problematic smartphone users is consistent with the results of previous studies on Internet gaming disorder (Weng et al., 2013) and Internet addiction (Hong et al., 2013; Lin \& Lei, 2015). Our results also are consistent with the results of a previous functional MRI study of problematic smartphone use, which identified alterations in functional connectivity in the OFC (Chun et al., 2018). Smaller GMV in the OFC was significantly correlated with higher SAPS score, reflecting a risk of addiction to online activities through smartphones. These results suggest that gray matter abnormalities in the OFC are involved in the pathophysiology of problematic smartphone use.

The OFC has been associated with reward-guided decision-making (Wallis, 2007). OFC abnormalities have been suggested to interfere with adaptive decision-making, thereby increasing impulsivity and forming stimulus-driven habits (Torregrossa, Quinn, \& Taylor, 2008). Dysfunctional decision-making and a progression from goal-directed actions into stimulus-driven habits have been associated with addiction (Bechara \& Damasio, 2002; Bechara, Dolan, \& Hindes, 2002). The OFC has been proposed to have a role as a neural correlate of the development and maintenance of addiction (Schoenbaum, Roesch, \& Stalnaker, 2006; Volkow \& Fowler, 2000). In particular, the cluster in the right OFC identified in this study corresponds to the lateral OFC, which is involved in suppressing behaviors that previously gave rewards and adjusting decisions to obtain delayed rewards (McClure, Laibson, Loewenstein, \& Cohen, 2004). We identified small GMV in the lateral OFC, which suggests that repeating habitual behaviors while pursuing immediate rewards would be implicated in problematic smartphone users. This study includes subjects who spend excessive amounts of time using social networking platforms via smartphones. Previous studies suggest that subjects with excessive social media use have difficulty in proper decision-making for the delayed reward (Delaney, Stein, \& Gruber, 2018; Turel, He, Brevers, \& Bechara, 2018).

This study correlated GMV in the lateral OFC with the SAPS tolerance subscale. In behavioral addiction, tolerance is defined as the increasing amount of time required for participation in particular activities to achieve the same levels of satisfaction (Griffiths, 1996). The SAPS tolerance subscale was calculated as the sum of the following items: "I try cutting my smartphone usage time, but I fail;" "I can control my smartphone usage time;" "Even when I think I should stop, I continue to use my smartphone;" and "Spending a lot of time on my smartphone has become a habit." These questionnaire items reflect an increase in the behavioral ranges of online smartphone activities, accompanied by loss of control over smartphone use. The lateral $\mathrm{OFC}$ is associated with the tendency to adaptively regulate control in response to negative consequences (O’Doherty, Critchley, Deichmann, \& Dolan, 2003). Neurobiological studies on substance addiction showed that disruption of the lateral OFC is associated with enhanced tolerance to the deleterious effects of substance use (Nimitvilai, Lopez, Mulholland, \& Woodward, 2016; 
Winstanley et al., 2007). To understand problematic smartphone use in terms of behavioral addiction, we infer that the lateral OFC may have a crucial role in forming the core manifestations of addiction.

We acknowledge several limitations of this study and points that require further discussion. First, there is no consensus on the terms used in this study. Although the results of this study were described from the perspective of behavioral addiction, the controversy regarding these terms is ongoing. The effects of media characteristics on excessive smartphone use and addictive potential of online activities are not well identified. The patterns of smartphone usage and related factors can vary, and numerous online activities and applications are available on the smartphone. Therefore, we used the phrase "problematic smartphone use" as a generic term, rather than using more specific terms such as "smartphone addiction" and "social networking addiction." The core characteristics of problematic smartphone use and the appropriate terminology to represent this psychiatric condition require further investigation and consensusbuilding. Second, this study was performed with a cross-sectional design, making it impossible to sufficiently discriminate between a predisposition to problematic smartphone use and the effects of long-term, uncontrolled, excessive smartphone use. A longitudinal follow-up study on Internet gaming disorder suggested that the reduction of GMV in the OFC might be due to excessive gaming (Zhou et al., 2019). Whether orbitofrontal gray matter abnormalities might result from excessive use of social networking platforms via smartphone requires further investigations. Third, the study relied on self-reported answers to questionnaires collecting information about clinical features associated with problematic smartphone use. Behavioral smartphone usage patterns obtained by the applications of the smartphone are needed to be explored further in future research. If neuropsychological tests evaluating executive control and decision-making are included in these future studies, a more reliable interpretation of neuroimaging results will be available.

\section{CONCLUSIONS}

In conclusion, this study identified characteristic lateral orbitofrontal gray matter abnormalities in subjects with problematic smartphone use. There were significant correlations between small GMV in the right lateral OFC and high SAPS score, particularly the SAPS tolerance subscale. The lateral $\mathrm{OFC}$ has important roles in reward-related decision-making and exercising appropriate regulatory control. We speculate that smartphone media characteristics, which provide continuous Internet connection, may function synergistically with lateral orbitofrontal gray matter abnormalities. This leads to increased amounts of time spent on smartphone online activities, accompanied by loss of control over smartphone use. Future studies on problematic smartphone use need to include a more objective measurement of smartphone usage patterns and clarify the aspects of problematic smartphone use that have potential risk of behavioral addiction.
Funding sources: This study was funded by a grant of the Korean Mental Health Technology R\&D Project, Ministry of Health and Welfare, Republic of Korea (HM14C2578).

Authors' contribution: DL and Y-CJ conceived and designed the study. DL and JL recruited participants. DL analyzed data and drafted the manuscript. $\mathrm{KN}$ and $\mathrm{BOL}$ provided critical revision of the manuscript and important intellectual content. All authors had full access to all data in the study and take responsibility for the integrity of the data and the accuracy of the data analysis. All authors critically reviewed and approved the final version of this manuscript for publication.

Conflict of interest: The authors declare no conflict of interest.

\section{REFERENCES}

Alemán-Gómez, Y. (2006, June 11-15). IBASPM: Toolbox for automatic parcellation of brain structures. Paper presented at the 12th Annual Meeting of the Organization for Human Brain Mapping, Florence, Italy.

Ashburner, J. (2007). A fast diffeomorphic image registration algorithm. Neuroimage, 38(1), 95-113. doi:10.1016/j.neuroimage. 2007.07.007

Ashburner, J. (2010). VBM tutorial (Technical report). London, UK: Wellcome Trust Centre for Neuroimaging.

Bechara, A., \& Damasio, H. (2002). Decision-making and addiction (part I): Impaired activation of somatic states in substance dependent individuals when pondering decisions with negative future consequences. Neuropsychologia, 40(10), 1675-1689. doi:10.1016/S0028-3932(02)00015-5

Bechara, A., Dolan, S., \& Hindes, A. (2002). Decision-making and addiction (part II): Myopia for the future or hypersensitivity to reward? Neuropsychologia, 40(10), 1690-1705. doi:10.1016/ S0028-3932(02)00016-7

Beck, A. T., Epstein, N., Brown, G., \& Steer, R. A. (1988). An inventory for measuring clinical anxiety: Psychometric properties. Journal of Consulting and Clinical Psychology, 56(6), 893-897. doi:10.1037/0022-006X.56.6.893

Beck, A. T., Steer, R. A., \& Brown, G. K. (1996). Beck Depression Inventory-II. San Antonio, 78, 490-498. doi:10.1037/t00742-000

Brand, M., Young, K. S., Laier, C., Wölfling, K., \& Potenza, M. N. (2016). Integrating psychological and neurobiological considerations regarding the development and maintenance of specific Internet-use disorders: An Interaction of PersonAffect-Cognition-Execution (I-PACE) model. Neuroscience and Biobehavioral Reviews, 71, 252-266. doi:10.1016/j. neubiorev.2016.08.033

Chen, J., Liang, Y., Mai, C., Zhong, X., \& Qu, C. (2016). General deficit in inhibitory control of excessive smartphone users: Evidence from an event-related potential study. Frontiers in Psychology, 7, 511. doi:10.3389/fpsyg.2016.00511

Chun, J.-W., Choi, J., Cho, H., Choi, M. R., Ahn, K. J., Choi, J. S., \& Kim, D. J. (2018). Role of frontostriatal connectivity in 
adolescents with excessive smartphone use. Frontiers in Psychiatry, 9, 437. doi:10.3389/fpsyt.2018.00437

Dalley, J. W., Cardinal, R. N., \& Robbins, T. W. (2004). Prefrontal executive and cognitive functions in rodents: Neural and neurochemical substrates. Neuroscience and Biobehavioral Reviews, 28(7), 771-784. doi:10.1016/j.neubiorev.2004.09.006

Delaney, D., Stein, L., \& Gruber, R. (2018). Facebook addiction and impulsive decision-making. Addiction Research \& Theory, 26(6), 478-486. doi:10.1080/16066359.2017.1406482

Dong, G., \& Potenza, M. N. (2014). A cognitive-behavioral model of Internet gaming disorder: Theoretical underpinnings and clinical implications. Journal of Psychiatric Research, 58, 7-11. doi:10.1016/j.jpsychires.2014.07.005

Elhai, J. D., Dvorak, R. D., Levine, J. C., \& Hall, B. J. (2017). Problematic smartphone use: A conceptual overview and systematic review of relations with anxiety and depression psychopathology. Journal of Affective Disorders, 207, 251-259. doi:10.1016/j.jad.2016.08.030

Elhai, J. D., Tiamiyu, M., \& Weeks, J. (2018). Depression and social anxiety in relation to problematic smartphone use: The prominent role of rumination. Internet Research, 28(2), 315-332. doi:10.1108/IntR-01-2017-0019

Everitt, B. J., Dickinson, A., \& Robbins, T. W. (2001). The neuropsychological basis of addictive behaviour. Brain Research Reviews, 36(2-3), 129-138. doi:10.1016/S01650173(01)00088-1

First, M. B., Spitzer, R. L., Gibbon, M., \& Williams, J. B. W. (1995). Structured clinical interview for DSM-IV axis I disorders. New York, NY: New York State Psychiatric Institute.

Friston, K. J., Worsley, K. J., Frackowiak, R. S., Mazziotta, J. C., \& Evans, A. C. (1994). Assessing the significance of focal activations using their spatial extent. Human Brain Mapping, 1(3), 210-220. doi:10.1002/hbm.460010306

Griffiths, M. (1996). Behavioural addiction: An issue for everybody? Employee Councelling Today, 8(3), 19-25. doi:10.1108/ 13665629610116872

Hadar, A., Hadas, I., Lazarovits, A., Alyagon, U., Eliraz, D., \& Zangen, A. (2017). Answering the missed call: Initial exploration of cognitive and electrophysiological changes associated with smartphone use and abuse. PLoS One, 12(7), e0180094. doi:10.1371/journal.pone.0180094

Hong, S.-B, Kim, J.-W, Choi, E.-J, Kim, H. H., Suh, J. E., Kim, C. D., Klauser, P., Whittle, S., Y cel, M., Pantelis, C., \& Yi, S. H. (2013). Reduced orbitofrontal cortical thickness in male adolescents with Internet addiction. Behavioral and Brain Functions, 9(1), 11. doi:10.1186/1744-9081-9-11

Jahanshahi, M., Obeso, I., Rothwell, J. C., \& Obeso, J. A. (2015). A fronto-striato-subthalamic-pallidal network for goaldirected and habitual inhibition. Nature Reviews Neuroscience, 16(12), 719-7322. doi:10.1038/nrn4038

Kim, D., Chung, Y., Lee, J., Kim, M. C., Lee, Y. H., Kang, E. B., Keum, C., \& Nam, J. (2012). Development of Smartphone Addiction Proneness Scale for adults: Self-report. The Korean Journal of Counseling, 29, 629-644. doi:10.15703/ kjc.13.2.201204.629

Kim, D., Lee, Y., Lee, J., Nam, J. K., \& Chung, Y. (2014). Development of Korean Smartphone Addiction Proneness Scale for youth. PLoS One, 9(5), e97920. doi:10.1371/ journal.pone.0097920

Lachmann, B., Sindermann, C., Sariyska, R. Y., Luo, R., Melchers, M. C., Becker, B., Cooper, A. J., \& Montag, C. (2018). The role of empathy and life satisfaction in Internet and smartphone use disorder. Frontiers in Psychology, 9, 398. doi:10.3389/ fpsyg.2018.00398

Lee, D., Namkoong, K., Lee, J., \& Jung, Y. C. (2018). Abnormal gray matter volume and impulsivity in young adults with Internet gaming disorder. Addiction Biology, 23(5), 1160-1167. doi:10.1111/adb.12552

Lin, F., \& Lei, H. (2015). Structural brain imaging and Internet addiction. In C. Montag \& M. Reuter (Eds.), Internet addiction (pp. 21-42). Cham, Switzerland: Springer.

Long, J., Liu, T.-Q, Liao, Y.-H, Qi, C., He, H. Y., Chen, S. B., \& Billieux, J. (2016). Prevalence and correlates of problematic smartphone use in a large random sample of Chinese undergraduates. BMC Psychiatry, 16(1), 408. doi:10.1186/ s12888-016-1083-3

Lopez-Fernandez, O., Honrubia-Serrano, L., Freixa-Blanxart, M., \& Gibson, W. (2014). Prevalence of problematic mobile phone use in British adolescents. Cyberpsychology, Behavior, and Social Networking, 17(2), 91-98. doi:10.1089/cyber.2012. 0260

Maldjian, J. A., Laurienti, P. J., Kraft, R. A., \& Burdette, J. H. (2003). An automated method for neuroanatomic and cytoarchitectonic atlas-based interrogation of fMRI data sets. Neuroimage, 19(3), 1233-1239. doi:10.1016/S1053-8119(03) 00169-1

McClure, S. M., Laibson, D. I., Loewenstein, G., \& Cohen, J. D. (2004). Separate neural systems value immediate and delayed monetary rewards. Science, 306(5695), 503-507. doi:10.1126/ science. 1100907

Miller, E., \& Wallis, J. (2009). Executive function and higher-order cognition: Definition and neural substrates. In L. R. Squire (Ed.), Encyclopedia of neuroscience (Vol. 4, pp. 99-104). Oxford, UK: Academic Press.

Montag, C., Zhao, Z., Sindermann, C., Xu, L., Fu, M., Li, J., Zheng, X., Li, K., Kendrick, K. M., Dai, J., \& Becker, B. (2018). Internet communication disorder and the structure of the human brain: Initial insights on WeChat addiction. Scientific Reports, 8(1), 2155. doi:10.1038/s41598-018-19904-y

Müller, J., Dreisbach, G., Goschke, T., Hensch, T., Lesch, K. P., \& Brocke, B. (2007). Dopamine and cognitive control: The prospect of monetary gains influences the balance between flexibility and stability in a set-shifting paradigm. European Journal of Neuroscience, 26(12), 3661-3668. doi:10.1111/ j.1460-9568.2007.05949.x

Nimitvilai, S., Lopez, M. F., Mulholland, P. J., \& Woodward, J. J. (2016). Chronic intermittent ethanol exposure enhances the excitability and synaptic plasticity of lateral orbitofrontal cortex neurons and induces a tolerance to the acute inhibitory actions of ethanol. Neuropsychopharmacology, 41(4), 1112-1127. doi:10.1038/npp.2015.250

O’Doherty, J., Critchley, H., Deichmann, R., \& Dolan, R. J. (2003). Dissociating valence of outcome from behavioral control in human orbital and ventral prefrontal cortices. Journal of Neuroscience, 23, 7931-7939. doi:10.1523/jneurosci.23-21-07931.2003

Patton, J. H., \& Stanford, M. S. (1995). Factor structure of the Barratt Impulsiveness Scale. Journal of Clinical Psychology, 51(6), 768-774. doi:10.1002/1097-4679(199511)51:6<768:: AID-JCLP2270510607>3.0.CO;2-1

Salehan, M., \& Negahban, A. (2013). Social networking on smartphones: When mobile phones become addictive. 
Computers in Human Behavior, 29(6), 2632-2639. doi:10.1016/j.chb.2013.07.003

Sapacz, M., Rockman, G., \& Clark, J. (2016). Are we addicted to our cell phones? Computers in Human Behavior, 57, 153-159. doi:10.1016/j.chb.2015.12.004

Saunders, J. B., Aasland, O. G., Babor, T. F., de la Fuente, J. R., \& Grant, M. (1993). Development of the Alcohol Use Disorders Identification Test (AUDIT): WHO collaborative project on early detection of persons with harmful alcohol consumptionII. Addiction, 88(6), 791-804. doi:10.1111/j.1360-0443. 1993.tb02093.x

Schoenbaum, G., Roesch, M. R., \& Stalnaker, T. A. (2006). Orbitofrontal cortex, decision-making and drug addiction. Trends in Neurosciences, 29(2), 116-124. doi:10.1016/ j.tins.2005.12.006

Sha, P., Sariyska, R., Riedl, R., Lachmann, B., \& Montag, C. (2019). Linking Internet communication and smartphone use disorder by taking a closer look at the Facebook and WhatsApp applications. Addictive Behaviors Reports, 9, 100148. doi:10.1016/j.abrep.2018.100148

Stald, G., Green, L., Barbovski, M., Haddon, L., Mascheroni, G., Sagvari, B., Scifo, B., \& Tsaliki, L. (2014). Online on the mobile: Internet use on smartphones and associated risks among youth in Europe. London, UK: EU Kids Online, LSE.

Torregrossa, M. M., Quinn, J. J., \& Taylor, J. R. (2008). Impulsivity, compulsivity, and habit: The role of orbitofrontal cortex revisited. Biological Psychiatry, 63(3), 253-255. doi:10.1016/j. biopsych.2007.11.014

Turel, O., He, Q., Brevers, D., \& Bechara, A. (2018). Delay discounting mediates the association between posterior insular cortex volume and social media addiction symptoms. Cognitive, Affective, \& Behavioral Neuroscience, 18(4), 694-704. doi:10.3758/s13415-018-0597-1

Turel, O., \& Serenko, A. (2012). The benefits and dangers of enjoyment with social networking websites. European Journal of Information Systems, 21(5), 512-528. doi:10.1057/ejis.2012.1

Volkow, N. D., \& Fowler, J. S. (2000). Addiction, a disease of compulsion and drive: Involvement of the orbitofrontal cortex. Cerebral Cortex, 10(3), 318-325. doi:10.1093/cercor/10.3.318

Wallis, J. D. (2007). Orbitofrontal cortex and its contribution to decision-making. Annual Review of Neuroscience, 30(1), 31-56. doi:10.1146/annurev.neuro.30.051606.094334

Wang, Y., Zou, Z., Song, H., Xu, X., Wang, H., d'Oleire Uquillas, F., \& Huang, X. (2016). Altered gray matter volume and white matter integrity in college students with mobile phone dependence. Frontiers in Psychology, 7, 597. doi:10.3389/ fpsyg.2016.00597

Wechsler, D. (2008). Wechsler Adult Intelligence Scale-Fourth Edition (WAIS-IV) (Vol. 22, p. 498). San Antonio, TX: NCS Pearson.

Wegmann, E., Stodt, B., \& Brand, M. (2018). Cue-induced craving in Internet-communication disorder using visual and auditory cues in a cue-reactivity paradigm. Addiction Research \& Theory, 26(4), 306-314. doi:10.1080/16066359.2017.1367385

Weng, C.-B., Qian, R.-B., Fu, X.-M, Lin, B., Han, X. P., Niu, C. S., \& Wang, Y. H. (2013). Gray matter and white matter abnormalities in online game addiction. European Journal of Radiology, 82(8), 1308-1312. doi:10.1016/j.ejrad.2013.01.031

Winstanley, C. A., LaPlant, Q., Theobald, D. E., Green, T. A., Bachtell, R. K., Perrotti, L. I., DiLeone, R. J., Russo, S. J., Garth, W. J., Self, D. W., \& Nestler, E. J. (2007). $\Delta$ FosB induction in orbitofrontal cortex mediates tolerance to cocaine-induced cognitive dysfunction. Journal of Neuroscience, 27(39), 10497-10507. doi:10.1523/JNEUROSCI.256607.2007

Wolniewicz, C. A., Tiamiyu, M. F., Weeks, J. W., \& Elhai, J. D. (2018). Problematic smartphone use and relations with negative affect, fear of missing out, and fear of negative and positive evaluation. Psychiatry Research, 262, 618-623. doi:10.1016/j.psychres.2017.09.058

Yao, Y.-W., Liu, L., Ma, S.-S., Shi, X. H., Zhou, N., Zhang, J. T., \& Potenza, M. N. (2017). Functional and structural neural alterations in Internet gaming disorder: A systematic review and meta-analysis. Neuroscience and Biobehavioral Reviews, 83, 313-324. doi:10.1016/j.neubiorev.2017.10.029

Young, K. S. (1998). Caught in the net: How to recognize the signs of Internet addiction-and a winning strategy for recovery. New York, NY: John Wiley \& Sons.

Yuan, P., \& Raz, N. (2014). Prefrontal cortex and executive functions in healthy adults: A meta-analysis of structural neuroimaging studies. Neuroscience and Biobehavioral Reviews, 42, 180-192. doi:10.1016/j.neubiorev.2014.02.005

Zhou, F., Montag, C., Sariyska, R., Lachmann, B., Reuter, M., Weber, B., Trautner, P., Kendrick, K. M., Markett, S., \& Becker, B. (2019). Orbitofrontal gray matter deficits as marker of Internet gaming disorder: Converging evidence from a cross-sectional and prospective longitudinal design. Addiction Biology, 24(1), 100-109. doi:10.1111/adb.12570 